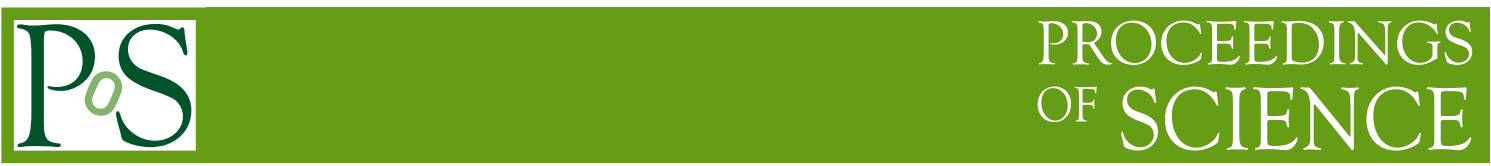

\title{
Charge Multiplication Properties in Highly Irradiated Epitaxial Silicon Detectors
}

\author{
Jörn Lange ${ }^{* a}$, Julian Becker ${ }^{a}$, Eckhart Fretwurst ${ }^{a}$, Robert Klanner ${ }^{a}$, Gregor \\ Kramberger $^{b}$, Gunnar Lindström ${ }^{a}$, Igor Mandić ${ }^{b}$ \\ ${ }^{a}$ Institute for Experimental Physics, University of Hamburg, Germany \\ ${ }^{b}$ Jožef Stefan Institute, Ljubljana, Slovenia \\ E-mail: joern. lange@desy.de
}

\begin{abstract}
Charge multiplication (CM) occuring in highly radiation-damaged Si sensors is currently under discussion as an option to overcome the strong trapping of charge carriers in the innermost pixel layers of future Super-LHC detectors. In this work, CM was studied in $\mathrm{p}^{+}-n$ epitaxial silicon pad diodes of 75, 100 and $150 \mu \mathrm{m}$ thickness after $24 \mathrm{GeV} / \mathrm{c}$ proton irradiation with $1 \mathrm{MeV}$ neutron equivalent fluences in the order of $10^{16} \mathrm{~cm}^{-2}$. Basic properties like the development and location of the CM region, proportionality between measured and deposited charge, spatial uniformity and long-term stability, which were studied with charge collection efficiency (CCE) measurements using the Transient Current Technique (TCT), are reviewed. Using ${ }^{90} \mathrm{Sr} \beta$-particles and an amplifier with $25 \mathrm{~ns}$ shaping time the signal in the $\mathrm{CM}$ regime for radiation similar to minimum ionising particles was investigated. Also associated noise, signal-to-noise ratio and effects of $\mathrm{CM}$ on the charge spectrum were studied.
\end{abstract}

19th International Workshop on Vertex Detectors

June 6 -11 2010

Loch Lomond, Scotland, UK

\footnotetext{
* Speaker.
} 


\section{Introduction}

The proposed upgrade of the LHC to a tenfold luminosity of $10^{35} \mathrm{~cm}^{-2} \mathrm{~s}^{-1}$ (Super-LHC or SLHC) will lead to a very harsh radiation environment for Si detectors in the tracking region [1]. With an expected $1 \mathrm{MeV}$ neutron equivalent fluence of $\Phi_{e q}=1.6 \times 10^{16} \mathrm{~cm}^{-2}$, the pixel sensors closest to the interaction point will be most affected. Thus silicon detectors as used today will suffer from severe radiation damage resulting in an increasing depletion voltage $\left(U_{d e p}\right)$ at high fluences, higher leakage currents $\left(I_{\text {rev }}\right)$ and trapping of charge carriers, leading to a strong degradation of the signal-to-noise ratio (SNR).

Trapping reduces the charge collection efficiency (CCE) significantly and is considered to be the most limiting factor at SLHC fluences. However, recently we observed CCE values larger than 1 in highly proton-irradiated thin epitaxial (EPI) diodes at high voltages [2, 3, 4]. This indicates that trapping can be overcompensated by charge multiplication (CM) due to impact ionisation at high electric fields after heavy irradiation. Evidence of CM leading to anomalously high charge collection has been also found in highly irradiated planar [5, 6, 7, 8] and 3D [9] strip detectors. Obviously, it would be desirable to use CM effects to overcome trapping in highly damaged $\mathrm{Si}$ sensors at the SLHC. Therefore, it is important to study the development and the properties of such a radiation-induced $\mathrm{CM}$ region in detail.

\section{Investigated material, samples and irradiation}

Epitaxial silicon combines defect engineering (high oxygen concentration) and device engineering (thin sensor layers) [10]. In this material, proton irradiation predominantly introduces donors, so that no space charge sign inversion (SCSI) occurs for n-type bulk silicon [11]. Moreover, the average electric field is higher in thin sensitive layers.

$\mathrm{N}$-type epitaxial layers of 75,100 and $150 \mu \mathrm{m}$ thickness and resistivities between 150 and $500 \Omega \mathrm{cm}$ were grown on a highly doped $\approx 500 \mu \mathrm{m}$ thick $\mathrm{Cz}$ substrate by ITME [12]. Both standard $\left(S T,[\mathrm{O}]=(5-9) \times 10^{16} \mathrm{~cm}^{-3}\right)$ and oxygen-enriched $\left(D O,[\mathrm{O}]=(1.4-6) \times 10^{17} \mathrm{~cm}^{-3}\right)$ materials were studied. $\mathrm{p}^{+}$-n pad diodes of $5 \times 5 \mathrm{~mm}^{2}$ (large) and $2.5 \times 2.5 \mathrm{~mm}^{2}$ (small) active area were processed at CiS [13]. The metallisation of the front side, where the p-n junction is located, has an optical window for illumination. If not stated otherwise, large diodes were used.

Irradiations with $24 \mathrm{GeV} / \mathrm{c}$ protons were performed at the CERN PS irradiation facility [14] with an average proton flux of several $10^{9} \mathrm{~cm}^{-2} \mathrm{~s}^{-1}$ at a temperature between 27 and $29^{\circ} \mathrm{C}$. Proton fluences were measured by $\mathrm{Al}$ foil activation with a quoted accuracy of $10 \%$ and scaled to $1 \mathrm{MeV}$ neutron equivalent fluences, which will be referred to in the following, using a hardness factor of $\kappa=0.62$. Prior to the measurements, the investigated samples were annealed for $30 \mathrm{~min}$ at $80^{\circ} \mathrm{C}$.

\section{Experimental methods}

Charge collection was investigated both with different Transient Current Technique (TCT) setups at Hamburg, using current-sensitive amplifiers and laser light or $\alpha$-particles, and with a ${ }^{90} \mathrm{Sr}$ $\beta$-setup at Ljubljana, using a charge-sensitive preamplifier with $25 \mathrm{~ns}$ shaping circuit. 


\subsection{Charge collection}

Mobile charge carriers produced by radiation drift in the electric field inside the sensor and induce a current at the electrodes that is proportional to their number, their drift velocities and the weighting field (in a pad diode simply the inverse of the sensor thickness $1 / d$ ) [15]. The number of carriers might decrease with time due to trapping, described by an effective trapping time $\tau_{\text {eff }}$ [16], or increase due to impact ionisation in high electric fields. Impact ionisation can be parameterised by ionisation coefficients $\alpha_{n, p}$ for electrons and holes $\left(\alpha_{n}>\alpha_{p}\right)$, which are a strong function of the electric field [17].

\subsection{Types of radiation}

In this work radiation with different penetration was used. For epitaxial diodes, only front illumination $\left(\mathrm{p}^{+}\right.$-side) is possible due to the thick $\mathrm{Cz}$ substrate at the back side. Red laser light of 660 or $670 \mathrm{~nm}$ wavelength $(\approx 3 \mu \mathrm{m}$ absorption length) produces charge carriers close to the illuminated front surface. Thus, holes are collected immediately at the adjacent $\mathrm{p}^{+}$-electrode and the current signal is almost entirely due to electrons drifting to the $\mathrm{n}^{+}$-back side. In contrast, $1060 \mathrm{~nm}$ laser light deposits charge almost uniformly over the whole detector thickness similarly to minimum ionising particles (MIPs) or $\beta$-particles. The penetration of $830 \mathrm{~nm}$ laser light with an absorption length of $\approx 13 \mu \mathrm{m}$ lies in between. $5.8 \mathrm{MeV} \alpha$-particles from a ${ }^{244} \mathrm{Cm}$ source feature a Bragg peak about $26 \mu \mathrm{m}$ deep inside the detector for perpendicular incidence. Polyethylene (PE) absorbers of different thicknesses $(12$ and $24 \mu \mathrm{m})$ can be inserted between the source and the sample, which shifts the Bragg peak closer to the surface.

\subsection{TCT setups}

With the TCT setups (see [4] for details), the transient current pulse was directly measured without shaping. Thus, the collected charge $Q$ could be determined as the integral of the current pulse and the CCE was obtained by normalising $Q$ to $Q_{0}$ of an unirradiated diode. The achieved CCE precision was estimated to be between 2 and $3 \%$.

The signal was read out at the front side using a bias-T, a $1.8 \mathrm{GHz}$ current-sensitive amplifier with a gain of 100 and an oscilloscope with 1 or $2.5 \mathrm{GHz}$ bandwidth. 512 current pulses were averaged and measurements were done at $-10^{\circ} \mathrm{C}$ in a nitrogen atmosphere.

Short laser light pulses ( $\approx 100$ ps for 660,670 and $1060 \mathrm{~nm} ; \approx 1 \mathrm{~ns}$ for $830 \mathrm{~nm}$ ) were guided via an optical fibre to the illumination window without additional focusing and typically a few $10^{6} \mathrm{e}-\mathrm{h}$ pairs were produced in the diode. The trigger for the oscilloscope was provided externally by the laser driver. For the measurements of $\alpha$-particles self-triggering was used.

For $\mathrm{x}$-y scans another setup with movable $\mathrm{x}$ - and $\mathrm{y}$-tables $(0.1 \mu \mathrm{m}$ precision $)$ was used. In this case, the light was focused to a measured spot size of $\sigma_{\text {spot }}=20 \mu \mathrm{m}$.

\section{$3.4 \beta$-setup}

In order to investigate the sensor response to MIP-like particles under realistic readout conditions, measurements with $\beta$-particles from a ${ }^{90} \mathrm{Sr}$ source using a charge-sensitive preamplifier and a shaping amplifier with $25 \mathrm{~ns}$ peaking time (LHC bunch crossing time) were performed. The setup is described in detail in [18]. The diodes were mounted in an Al box, which was placed on an Al 

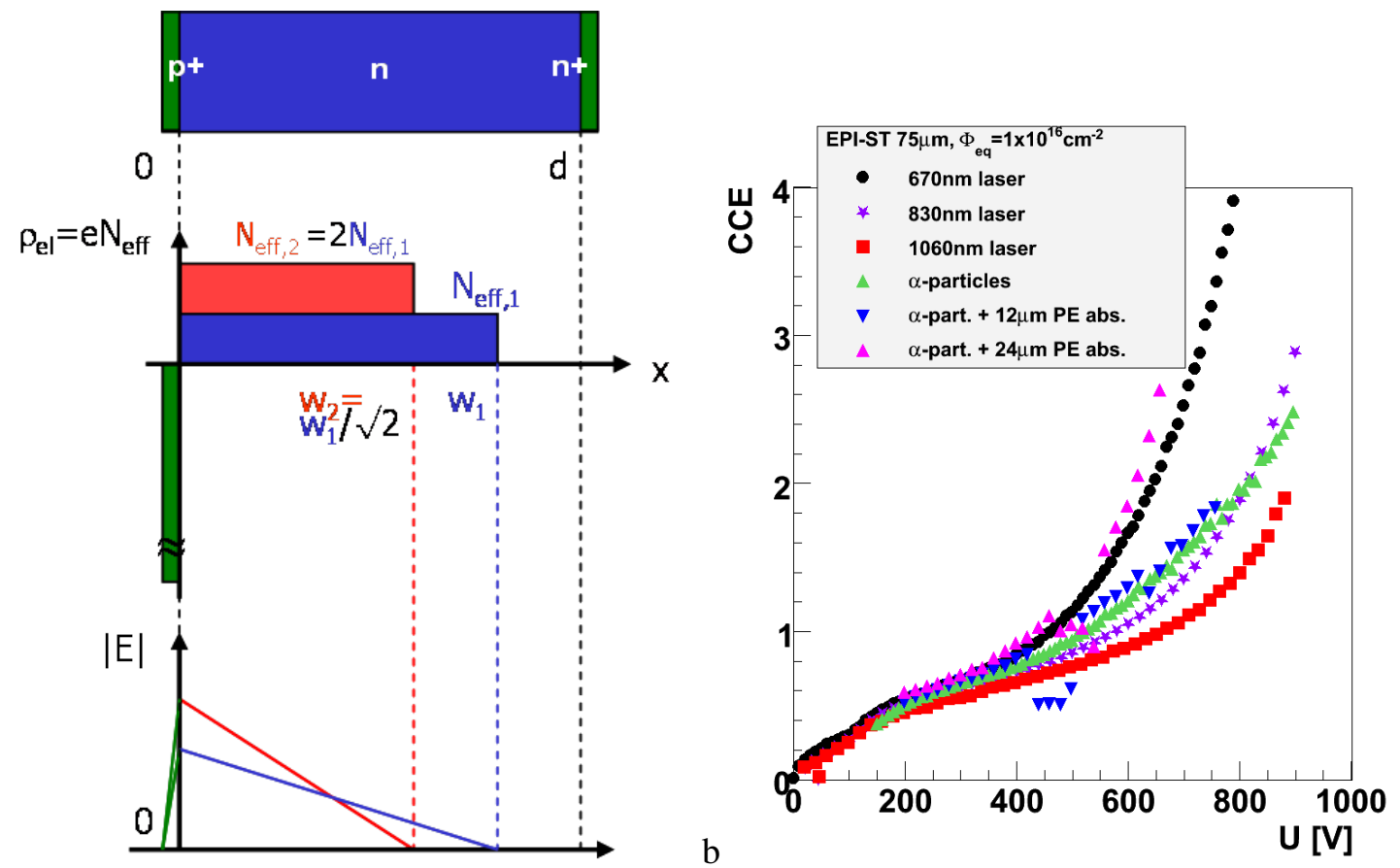

Figure 1: a) Sketch of the diode (top), two different uniform space charge distributions $\rho_{e l}(x)$, where $N_{\text {eff }}$ is the effective doping concentration and $w$ the width of the space charge region (middle) and the resulting linear electric field distributions $E(x)$ (bottom). b) The CCE as a function of bias voltage measured with different types of radiation. The CCE of $670 \mathrm{~nm}$ laser light increases to values above 7 at $900 \mathrm{~V}$. Low CCE values in certain voltage ranges for $\alpha$-particles with absorbers are due to triggering by micro discharges.

support plate that was cooled to $\approx-29^{\circ} \mathrm{C}$ by Peltier elements. Small holes in the lid and bottom of the box and in the plate served as a collimator for the $\beta$-particles. A scintillator under the cold plate provided a trigger with high purity ( $>97 \%$ ), which enabled measurements also at low SNR. The signal pulse was recorded by a digital oscilloscope and analysed offline. At least 2500 signals were measured, for low SNR up to 7500. The pulse height-to-charge calibration was performed with $59.5 \mathrm{keV}$ photons from an ${ }^{241} \mathrm{Am}$ source using an unirradiated diode.

\section{Development and properties of the CM region measured with TCT}

The properties of the CM region as measured with the TCT setups with laser light and $\alpha$ particles are reviewed. For a more detailed elaboration see [4]. If not stated otherwise, measurements were performed with red laser light on an EPI-ST $75 \mu \mathrm{m}$ diode irradiated to $10^{16} \mathrm{~cm}^{-2}$.

\subsection{Development and localisation of the CM region}

To understand the development of a radiation-induced CM region, a simplified model for the change of the electric field due to radiation-induced space charge is considered. Fig. 1a shows the resulting electric fields for two different space charge distributions. For a uniform space charge a linear electric field distribution with the maximum at the $p-n$ junction is obtained. As mentioned in Sec. 2, proton irradiation of EPI material introduces predominantly donors (no SCSI), so that the 
positive space charge density increases. This results in a steeper slope of the electric field, which leads to a higher maximum field value at the junction. Thus, if a sufficiently high density of donors is introduced at high fluences, the electric field in a layer around the junction is expected to become sufficiently large for impact ionisation at high voltages. It should be noted that the actual shape of the electric field distribution in highly irradiated diodes deviates substantially from linear [20, 21]: The high level of reverse current modifies the space charge distribution by changing the occupation of defects, leading to a double junction, and it causes a voltage drop over the neutral bulk region which becomes highly resistive after irradiation.

Measurements using radiation with different penetration have shown a stronger CM if charge carriers are produced closer to the front surface (see Fig. 1b), thereby confirming the existence of a thin $\mathrm{CM}$ region near the $\mathrm{p}^{+}$-implant.

\subsection{Linearity of multiplication}

The measured integrated charge $Q$ was found to be proportional to the deposited charge $Q_{0}$, also in the CM regime. For Geiger mode, which would result in signal saturation, a substantial multiplication contribution of holes and therefore higher electric fields would be needed.

\subsection{Spatial uniformity over the diode area}

$\mathrm{x}-\mathrm{y}$ scans over the illumination window in the front metallisation of the diode were performed with a laser spot focused to $\sigma_{\text {spot }}=20 \mu \mathrm{m}$ and a step width of $200 \mu \mathrm{m}$ at different voltages in the $\mathrm{CM}$ regime. In all cases, the CCE was found to be uniform over the whole area with deviations on the sub-percent level.

\subsection{Long-term stability}

To operate a real detector in the $\mathrm{CM}$ regime, its gain and noise have to remain stable over a long period of time. Repeated measurements at a constant bias voltage of $480 \mathrm{~V}$ and $900 \mathrm{~V}$ over 7 and 4 days, respectively, show that the collected charge is constant within $10 \%$. However, at $900 \mathrm{~V}$ micro discharges occurred, the frequency of which increased substantially after 2.5 days. Thus, for operation at such high voltages, micro discharges need to be avoided.

\section{Charge collection with a ${ }^{90} \mathrm{Sr} \beta$-setup}

From the height of the signal pulses, charge spectra were obtained and fitted with a convolution of a Landau ${ }^{1}$ and a Gaussian distribution in order to determine the most probable value (MPV) of the collected charge. However, due to the strong noise increase, the MPV of the Landau component could not be determined anymore at high fluences and voltages. But averaging the signal pulses decreases the noise dramatically, so that the determination of the mean collected charge via the pulse height of the averaged signal was still reliably possible.

\subsection{Collected charge before irradiation}

As $\beta$-particles traverse the sensor, the deposited charge depends on the thickness. Fig. 2 shows that the MPV and the mean collected charge increase linearly as a function of thickness

\footnotetext{
${ }^{1}$ As defined in the ROOT [19] library.
} 


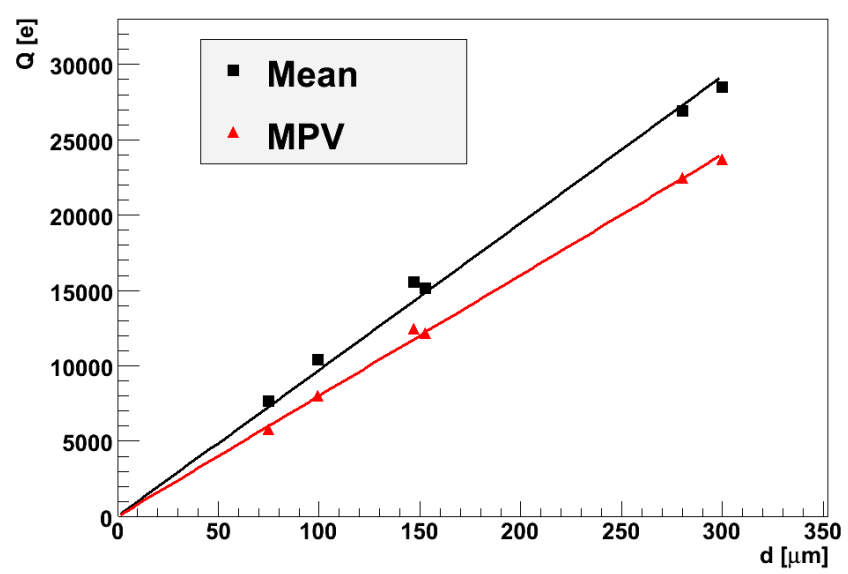

Figure 2: Mean and most probable collected charge for $\beta$-particles as a function of detector thickness before irradiation. FZ material was used for the 280 and $300 \mu \mathrm{m}$ thick diodes. For $150 \mu \mathrm{m}$, two diodes (small and large) are compared. a
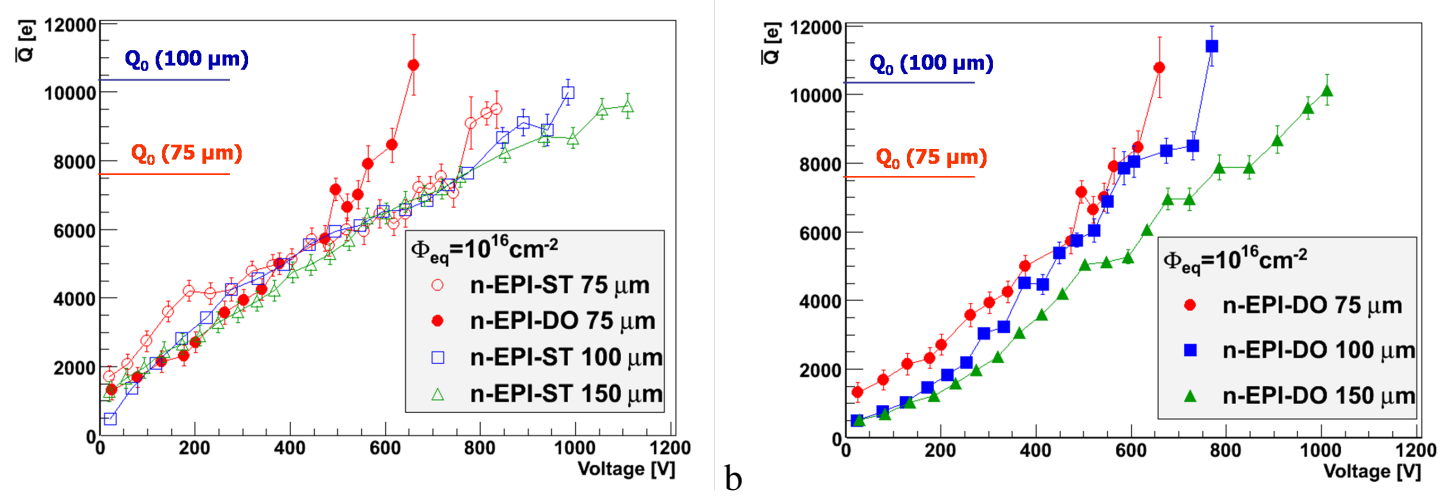

Figure 3: Mean collected charge for $\beta$-particles as a function of voltage for diodes irradiated to $10^{16} \mathrm{~cm}^{-2}$ for ST (a) and DO (b) material. For easier comparison of ST and DO diodes, the curve for EPI-DO $75 \mu \mathrm{m}$ was also included in a). The horizontal lines indicate the collected charge before irradiation, $Q_{0}$.

before irradiation. The slope was determined by a linear fit as 80 e-h pairs/ $\mu \mathrm{m}$ (MPV) and 97 e-h pairs/ $\mu \mathrm{m}$ (mean), respectively, which is in perfect agreement with measurements of 25 to $75 \mu \mathrm{m}$ thick epitaxial diodes [18]. Thus, the ratio between MPV and mean is around 0.8. The noise before irradiation was measured between 2000 and 3300 e depending on diode size and thickness.

\subsection{Collected charge after irradiation}

Fig. 3 shows the mean collected charge for $\beta$-particles as a function of voltage for diodes irradiated to $10^{16} \mathrm{~cm}^{-2}$. From the comparison of different thicknesses it can be seen that similar or even more charge is collected in thinner diodes compared to thicker ones although less charge is deposited. This can be explained by the higher values of both electric field (average value U/d) and weighting field (1/d) in thinner diodes. Higher electric fields lead to stronger CM at high voltages and to a faster drift at low voltages (below the saturation velocity regime), which increases 

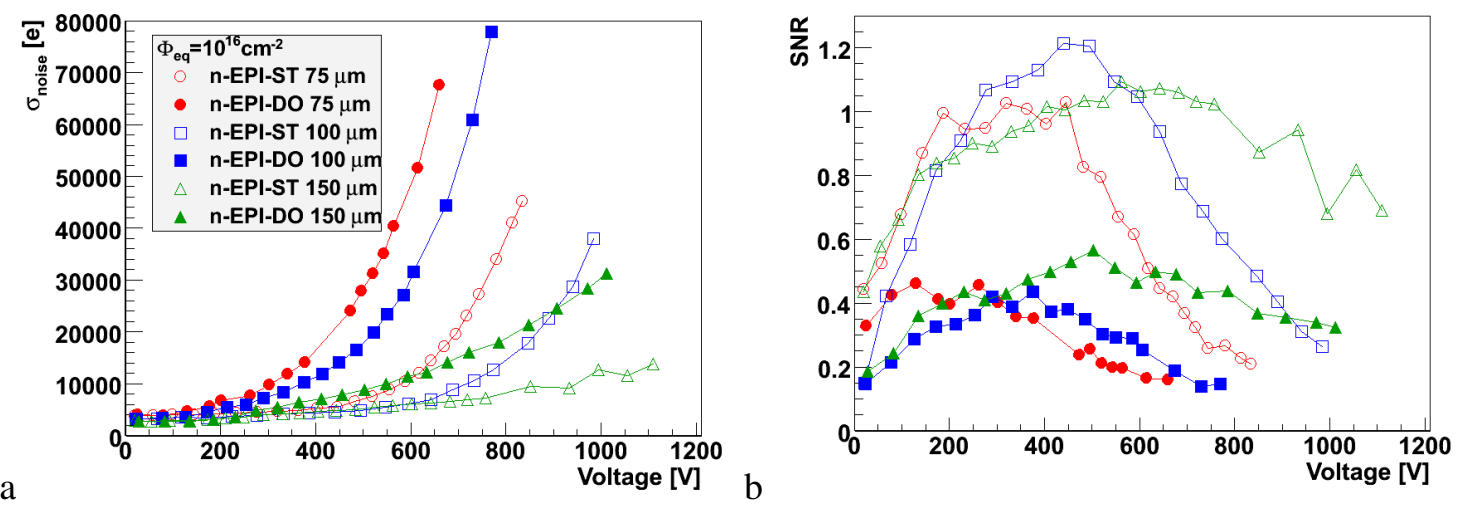

Figure 4: Noise (a) and mean SNR (b) as a function of applied voltage at a fluence of $10^{16} \mathrm{~cm}^{-2}$ for all investigated thicknesses and materials.

the traversed distance of charge carriers before being trapped. Moreover, even for the same travelled distance, the measured charge per carrier will be higher in thinner diodes due to the higher weighting field.

It can be seen that at low voltages (below the $\mathrm{CM}$ regime) the collected charge is lower for DO diodes than for ST material, whereas it is the other way round at high voltages (in the CM regime). Both effects can be explained by a higher donor introduction rate in DO material $[2,11]$, resulting in a higher space charge density. For DO diodes, this leads to a smaller depleted (i.e. sensitive) region at low voltages, whereas at high voltages the larger maximum field value at the p-n junction causes a stronger CM.

The dependence of $\mathrm{CM}$ on material and thickness observed here for $\beta$-particles is consistent with results for red laser light [4].

To conclude, very high values of collected charge were measured at SLHC fluences of $10^{16} \mathrm{~cm}^{-2}$. For all investigated materials and thicknesses, more than $9000 \mathrm{e}^{2}$ were measured ${ }^{2}$ at high voltages for $\beta$-particles. Thus, for $75 \mu \mathrm{m}$ diodes the signal clearly exceeds the pre-irradiation values and for $100 \mu \mathrm{m}$ diodes, at the maximum voltage measured, about the same charge is collected than before irradiation. Even at $500 \mathrm{~V}$, which is considered the voltage limit for today's LHC detectors, in all cases a signal of more than 5000 e could be achieved, which are remarkable values.

\subsection{Noise and signal-to-noise-ratio}

Due to impact ionisation, not only the signal is multiplied, but also the bulk reverse current:

$$
I_{\text {rev }}\left(M^{\prime}\right)=M^{\prime} I_{r e v, M^{\prime}=1},
$$

where $M^{\prime}$ is a multiplication factor that might differ from $M$ for the signal. Thus, also the shot noise $^{3} \sigma_{s h}$ (parallel or current noise) is expected to increase. In the absence of $\mathrm{CM}$, it is given by $\sigma_{s h}=\sqrt{2 e I_{\text {rev }} A_{s} \tau_{s}}$, where $e$ is the elementary charge, $A_{s}$ a shaper-dependent form factor and $\tau_{s}$ the shaping time. It should be noted that the shot noise in the CM regime cannot be obtained by

\footnotetext{
${ }^{2}$ Note that these are mean values, which can be roughly converted to MPVs by scaling with 0.8 .

${ }^{3}$ Noise is given in units of equivalent noise charge (ENC).
} 
inserting the multiplied reverse current (Eq. 5.1) into this formula, which would give $\sigma_{s h}\left(M^{\prime}\right)=$ $\sqrt{M^{\prime}} \sigma_{s h, M^{\prime}=1}$, where $\sigma_{s h, M^{\prime}=1}=\sqrt{2 e I_{r e v, M^{\prime}=1} A_{s} \tau_{s}}$ is the shot noise without multiplication. Instead, $\sigma_{s h, M^{\prime}=1}$ is multiplied directly by $M^{\prime}$ and might be further enhanced by statistical fluctuations in the $\mathrm{CM}$ process, described by the excess noise factor $F^{\prime}$ [22]:

$$
\sigma_{s h}\left(M^{\prime}\right)=M^{\prime} \sqrt{F^{\prime}} \sigma_{s h, M^{\prime}=1} .
$$

The total noise is the sum of this multiplication-dependent shot noise and noise contributions $\sigma_{\text {noise }}^{\prime}$ which are not influenced by CM (e.g. serial or voltage noise). Thus, the SNR is given by

$$
S N R=\frac{\bar{Q}}{\sigma_{\text {noise }}}=\frac{M \bar{Q}_{M=1}}{\sqrt{M^{\prime 2} F^{\prime} \sigma_{s h, M^{\prime}=1}^{2}+\sigma_{\text {noise }}^{\prime 2}}} .
$$

It can be seen that it depends on the relative size of the different terms whether CM can improve the SNR.

For measurements of EPI-ST $75 \mu \mathrm{m}$ irradiated to $10^{16} \mathrm{~cm}^{-2}$ with the TCT setup and laser light, an improvement of SNR in the CM regime up to the maximum measured voltage of $900 \mathrm{~V}$ was observed [4]. As the multiplication-independent intrinsic noise of the setup is large ( $\approx 25000 \mathrm{e})$, it dominates at moderate voltages over the shot noise which increases due to $\mathrm{CM}$, but starts from a much lower level. Thus the total noise remains about constant up to $\approx 700 \mathrm{~V}$. Then the shot noise begins to dominate, leading to a total noise increase to $\approx 45000$ e at $900 \mathrm{~V}$. But as the shot noise is still in the transition phase of being at the same order of magnitude as the intrinsic noise, the signal still increases faster over this range, so that the SNR improves.

Fig. 4 shows that the situation is different for the $\beta$-setup as its intrinsic noise is only between 2000 and 3000 e. Therefore, the shot noise begins to dominate already at much lower voltages, which leads to a fast total noise increase. Thus, after an increase of SNR at low voltages, SNR reaches a maximum between 300 and $500 \mathrm{~V}$, before decreasing (see Fig. 4b). Apparently, at high voltages the noise increases faster than the signal. The material and thickness dependence is the same as for the signal: Due to higher CM, the noise at high voltages is higher for DO compared to ST material and for thinner diodes compared to thicker ones. For the SNR, it is the other way round.

The strong noise increase, in certain cases up to 70000 e, leads to SNRs below 1. It is clear that detectors cannot be operated under such conditions. However, from Eq. 5.3 it follows that every setup and device has to be considered individually, as demonstrated by comparing the TCT and $\beta$-setups. Due to the smaller cell size, the reverse current in pixel sensors is much lower than in pad diodes, which reduces the shot noise (with and without $\mathrm{CM}$ ) while the signal stays the same (neglecting charge sharing). Decreasing the temperature might be another option to reduce the noise. Moreover, for the operation of pixel detectors, the signal threshold is usually set much higher than the noise due to reasons not related to the sensor noise (e.g. cross talk on readout chips). Therefore, increase of noise due to CM might be tolerable within certain limits.

\subsection{Charge spectrum}

For an efficient separation of signal and noise, it is not enough to consider the mean SNR, but the whole charge spectrum and its overlap with the noise spectrum must be taken into account. 


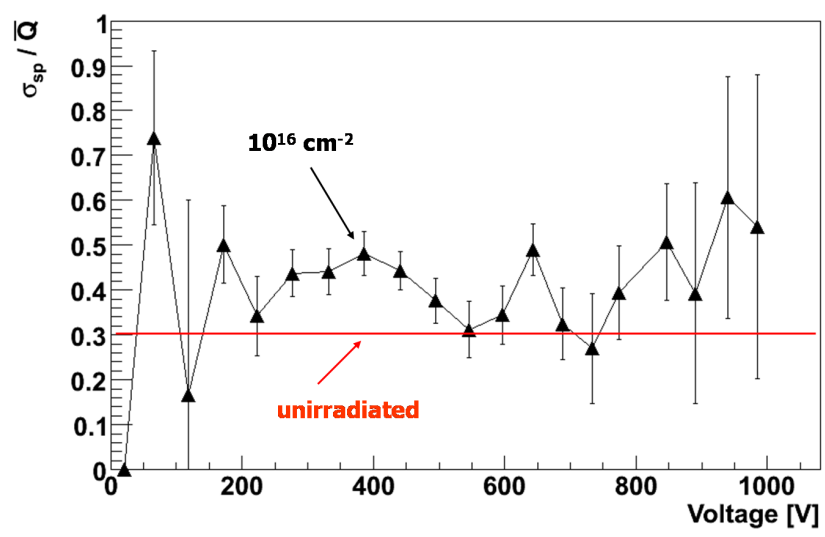

Figure 5: The noise-corrected relative width of the charge spectrum of EPI-ST $100 \mu \mathrm{m}$ irradiated to $10^{16} \mathrm{~cm}^{-2}$. The line indicates the level before irradiation.

Therefore, the charge spectrum must not broaden too much. However, its width is expected to increase due to multiplication and associated statistical fluctuations in a similar way as the shot noise (but note the different $M$ and $F$ ): $\sigma_{s p}=M \sqrt{F} \sigma_{s p, M=1}$. Moreover, Landau fluctuations of the amount of charge deposited in the thin CM region might additionally broaden the charge spectrum.

However, the charge spectrum can only be measured convoluted with the noise, so that the measured width $\left(\sigma_{s p, \text { meas }}\right.$ obtained by a Gaussian fit) must be corrected: $\sigma_{s p}=\sqrt{\sigma_{s p \text {,meas }}^{2}-\sigma_{\text {noise }}^{2}}$. Thus, large errors are introduced as the noise dominates the spectrum in the CM regime. Nevertheless, it can be seen from Fig. 5 for the example of an EPI-ST $100 \mu \mathrm{m}$ diode that the relative width ${ }^{4}$ $\sigma_{s p} / \bar{Q}$ does not increase significantly at high voltages in the CM regime. It is not much higher than the relative width before irradiation. To conclude, no significant impact of fluctuations in the $\mathrm{CM}$ process or the deposited charge in the CM region on the spectrum is observed. This suggests that $F$ is close to 1 , which is expected for moderate $\mathrm{CM}$. The same was observed for measurements with laser light [4].

\section{Conclusions}

Charge multiplication (CM) in thin epitaxial n-type pad diodes irradiated by protons up to $10^{16} \mathrm{~cm}^{-2}$ equivalent fluence was investigated by charge collection measurements with different TCT and $\beta$-setups. The CM region could be localised near the $\mathrm{p}^{+}$-implant at the front side. Observed properties like proportionality between measured and deposited charge, spatial uniformity over the diode area and long-term stability are promising for the operation of detectors in the CM regime. Large signals due to $\mathrm{CM}$ were also observed for $\beta$-particles. Higher fields in thin sensors and DO material lead to higher collected charges in the CM regime. However, at high voltages the noise was observed to increase faster than the signal leading to a degradation of the SNR. No influence of CM fluctuations on the charge spectrum was observed.

It was shown that high signals are possible at SLHC fluences due to CM. However, it must be investigated whether the noise can be better controlled in segmented devices than in pad diodes due

\footnotetext{
${ }^{4} \mathrm{By}$ normalising $\sigma_{s p}$ to $\bar{Q}$ the dependence on $\mathrm{M}$ cancels, so that the relative width is only sensitive to fluctuations.
} 
to their smaller reverse current and capacitance. Moreover, for segmented detectors the uniformity must be studied again as the bending of field lines at the implants could lead to non-uniform CM. Thus, effects of CM on the position resolution need to be investigated as well.

\section{Acknowledgements}

This work was performed in the frame of the CERN-RD50 collaboration. We would like to thank M. Glaser for his help with the proton irradiations. Partial funding by the German Ministry of Education and Research (BMBF) under the project "FSP 102 - CMS Detektor am LHC", by the HGF Alliance "Physics at the Terascale", by the CiS Hamburg project under Contract no. SSD 0517/03/05 and by the European XFEL is gratefully acknowledged.

\section{References}

[1] F. Gianotti et al., hep-ph/02004087, April 2002.

[2] J. Lange, Radiation Damage in Proton-Irradiated Epitaxial Silicon Detectors, Diploma thesis, University of Hamburg, October 2008, DESY-THESIS-2009-022.

[3] J. Lange et al., Nucl. Instr. and Meth. A (2010), doi:10.1016/j.nima.2009.11.082.

[4] J. Lange et al., Nucl. Instr. and Meth. A 622 (2010) 49.

[5] I. Mandić et al., Nucl. Instr. and Meth. A 603 (2009) 263.

[6] G. Kramberger et al., IEEE NSS Conference Record, N25-206, Orlando, USA, 2009.

[7] M. Mikuž et al., Nucl. Instr. and Meth. A (2010), doi:10.1016/j.nima.2010.04.084.

[8] G. Casse et al., Nucl. Instr. and Meth. A (2010), doi:10.1016/j.nima.2010.02.134.

[9] M. Köhler et al., Test Beam and Laser Measurements With Irradiated 3D Silicon Strip Detectors, presented at the 16th RD50 Workshop, Barcelona, June 2010,

http://indico.cern.ch/getFile.py/access?contribId=30\&sessionId=6\&resId=0\&materialId= slides\& confId $=86625$.

[10] G. Lindström et al., Nucl. Instr. and Meth. A 568 (2006) 66.

[11] I. Pintilie et al., Nucl. Instr. and Meth. A 611 (2009) 52.

[12] Institute of Electronic Materials Technology (ITME), 133 Wolczynska Str., 01-919 Warsaw, Poland.

[13] Forschungsinstitut für Mikrosensorik und Photovoltaik GmbH (CiS), Konrad-Zuse-Str. 14, 99099 Erfurt, Germany.

[14] M. Glaser. http://irradiation.web.cern.ch/irradiation.

[15] S. Ramo, Proc. IRE 27 (1939) 584.

[16] G. Kramberger et al., Nucl. Instr. and Meth. A 481 (2002) 297.

[17] W. N. Grant, Solid-St. Electron. 16 (1973) 1189.

[18] G. Kramberger et al., Nucl. Instr. and Meth. A 554 (2005) 212.

[19] ROOT. A Data Analysis Framework. http://root.cern.ch.

[20] V. Eremin et al., Nucl. Instr. and Meth. A 476 (2002) 556.

[21] E. Verbitskaya et al., Nucl. Instr. and Meth. A 583 (2007) 77.

[22] P. P. Webb et al., RCA Review 35 (1974) 234. 